\title{
Comparison of same day diagnostic tools including Gene Xpert and unstimulated IFN- $\gamma$ for the evaluation of pleural tuberculosis: a prospective cohort study
}

Richard Meldau', Jonny Peter', Grant Theron', Greg Calligaro ${ }^{1}$, Brian Allwood', Greg Symons ${ }^{1}$, Hoosain Khalfey ${ }^{1}$, Gina Ntombenhle ${ }^{1}$, Ureshnie Govender ${ }^{1}$, Anke Binder ${ }^{1}$, Richard van Zyl-Smit ${ }^{1}$ and Keertan Dheda ${ }^{1,2^{*}}$

\begin{abstract}
Background: The accuracy of currently available same-day diagnostic tools (smear microscopy and conventional nucleic acid amplification tests) for pleural tuberculosis (TB) is sub-optimal. Newer technologies may offer improved detection.

Methods: Smear-microscopy, adenosine deaminase (ADA), interferon gamma (IFN- $\gamma$ ), and Xpert MTB/RIF [using an unprocessed $(1 \mathrm{ml})$ and centrifuged $(\sim 20 \mathrm{ml})$ sample] test accuracy was evaluated in pleural fluid from 103 consecutive patients with suspected pleural TB. Culture for M.tuberculosis and/or histopathology (pleural biopsy) served as the reference standard. Patients were followed prospectively to determine their diagnostic categorisation.

Results: Of 93 evaluable participants, 40 had definite-TB (reference positive), 5 probable-TB (not definite but treated for TB) and 48 non-TB (culture and histology negative, and not treated for TB). Xpert MTB/RIF sensitivity and specificity (95\% Cl) was $22.5 \%$ (12.4 - 37.6) and 98\% (89.2 - 99.7), respectively, and centrifugation did not improve sensitivity (23.7\%). The Xpert MTB/RIF internal positive control showed no evidence of inhibition. Biomarker specific sensitivity, specificity, PPV, and NPVs were: ADA (48.85 IU/L; rule-in cut-point) 55.3\% (39.8 - 69.9), 95.2\% (83.9 - 98.7), 91.4 (73.4 - 95.4), 69.7\% (56.7 - 80.1); ADA (30 IU/L; clinically used cut-point) 79\% (63.7 - 89), 92.7\% (80.6 - 97.5), 91.0 (73.4 - 95.4), 82.7\% (69.3 - 90.1); and IFN- $\gamma$ (107.7 pg/ml; rule-in cut-point) 92.5\% (80.2 - 97.5), 95.9\% (86.1 - 98.9), 94.9\% (83.2 - 98.6), 93.9\% (83.5 - 97.9), respectively (IFN- $\gamma$ sensitivity and NPV better than Xpert $[p<0.05]$ and rule-in ADA $[p<0.05])$.

Conclusion: The usefulness of Xpert MTB/RIF to diagnose pleural TB is limited by its poor sensitivity. IFN- $\gamma$ is an excellent rule-in test and, compared to ADA, has significantly better sensitivity and rule-out value in a TB-endemic setting.
\end{abstract}

Keywords: Tuberculosis, Diagnosis, Xpert MTB/RIF, Interferon gamma, Adenosine deaminase, Pleural fluid

\section{Background}

Tuberculosis (TB) remains a global health problem, with an estimated 1.4 million deaths and 8.7 million new cases reported in 2011 [1]. Pulmonary TB is the most common form of TB, with extrapulmonary TB (EPTB) accounting for $\sim 15 \%$ of cases, but this estimate increases to $\sim 50 \%$ in high HIV prevalence settings [2]. Pleural TB,

\footnotetext{
*Correspondence: keertan.dheda@uct.ac.za

'Lung Infection and Immunity Unit, Division of Pulmonology \& UCT Lung Institute, Department of Medicine, University of Cape Town, Cape Town, South Africa

${ }^{2}$ Institute of Infectious Diseases and Molecular Medicine, University of Cape

Town, Cape Town, South Africa
}

a common form of EPTB, remains a common problem for physicians practising in high, intermediate and low TB burden settings, particularly where there are large immigrant populations. The diagnosis of pleural TB is challenging due to the paucibacillary nature of biological samples, and the need for diagnostic confirmation using invasive, expensive, and time consuming procedures such as blind pleural biopsy, imaging-guided-pleural biopsy, and medical or surgical thoracoscopy [3].

Proxy markers such as adenosine deaminase (ADA), an enzyme that catalyzes the conversion of adenosine and deoxyadenosine to inosine and deoxyinosine has

\section{Ciomed Central}


been widely studied [4]. ADA testing is relatively easy, inexpensive and rapid, with pooled sensitivity and specificity estimates of $92 \%$ and $90 \%$, respectively, across different prevalence settings depending on the cut-point used [4]. Interferon gamma (IFN- $\gamma$ ), an inflammatory cytokine secreted from macrophages and CD4 (+) T cells in response to $M$. tuberculosis infection, and that tends to concentrate in the pleural space, has been shown to be an alternative biological marker for pleural TB diagnosis with pooled sensitivity and specificity estimates of $89 \%$ and $97 \%$, respectively [5]. In high TB/HIV burden settings the performance was shown to be even better: sensitivity $97 \%$ and specificity $100 \%$ [6].

More recently the Xpert MTB/RIF assay, a fully automated quantitative real-time hemi-nested PCR, was introduced into high burden settings and is able to detect M. tuberculosis within 2 hours and also provide information about rifampicin susceptibility [7]. The assay has been validated using sputum samples and recently endorsed by the WHO as a rapid test for both smearpositive and smear-negative (paucibacillary) respiratory samples $[8,9]$. However, there are limited data about the Xpert MTB/RIF assay using pleural fluid [10-17] and thus the usefulness of this assay in the context of pleural $\mathrm{TB}$ remains unclear. Limitations of previously published work include the relatively small number of patients with pleural TB (usually quoted as part of a larger series of patients with EPTB), a paucity of biopsy-proven or culture positive samples as a gold standard, the lack of comparative analysis with other commonly used biomarkers, and a lack of attention to the technical factors that could impact Xpert MTB/RIF performance, including PCR inhibition, level of detection, and correlation with bacterial load. Furthermore, there are also limited data from high TB and HIV prevalence settings.

To address these knowledge gaps we prospectively evaluated the performance of the Xpert MTB/RIF assay, and other same-day diagnostic biomarkers, using pleural fluid obtained from patients with suspected pleural TB from Cape Town in South Africa.

\section{Methods \\ Patient recruitment, characterization and routine laboratory tests}

Consecutive patients with suspected pleural TB, including any symptoms including cough, fever, night sweats, loss of weight, haemoptysis and chest pain, and features consistent with a pleural effusion on chest $\mathrm{x}$-ray, were prospectively recruited from Groote Schuur, Somerset and Victoria Hospitals in Cape Town, South Africa, over a three year period from October 2009 to September 2012. The University of Cape Town Human Research Ethics Committee approved the study, and all patients provided written informed consent for study participation and pleural biopsy.

Routine TB diagnostic work up (pleural fluid analysis, sputum for microscopy and culture, when available, and lymph node or other organ biopsy) was performed by the referring physician. Although not routine, a closed pleural biopsy using an Abrams needle was performed by a study physician trained in this procedure to improve patient categorization. All biopsies were performed after aspiration of pleural fluid. Patients were offered voluntary HIV testing. Pleural fluid samples were collected for routine biochemical and cytological analysis (protein, albumin, ADA, glucose, cell differential, cytology), concentrated fluorescence smear microscopy, and liquid culture for M. tuberculosis using the MGIT 960 (Becton Dickinson, Sparks, Maryland) with the remaining fluid used for Xpert MTB/RIF and IFN- $\gamma$ analysis. Pleural biopsy samples were sent for histology and liquid culture. Adenosine deaminase activity in pleural fluid was determined by colorimetric technique by the National Health Laboratory Services, using the userdefined method on a Roche Cobas Integra (Roche Diagnostics Ltd, Switzerland). Pleural fluid ADA levels greater than $30 \mathrm{U} / \mathrm{L}$, in keeping with local guidelines and clinical practice, were reported as suggestive of pleural TB $[18,19]$.

Given the limitations of a single pleural fluid TB culture for confirming a diagnosis, patients were categorised as follows: Definite-TB: patients with at least one positive M. tuberculosis culture by liquid culture (in either pleural fluid, biopsy or sputum) and/or caseating granulomatous inflammation suggestive of TB on histological examination of pleural biopsy tissue, and with improvement on anti-TB treatment (all patients in this category received anti-TB treatment); Probable-TB: patients not meeting the criteria for definite-TB but with a clinical-radiological picture suggestive of $\mathrm{TB}$ and who were treated for TB with clinical response (all patients in this category received anti-TB treatment); Non-TB: patients for whom no microbiological or histological evidence of $M$. tuberculosis could be found, and/or for whom an alternative diagnosis was available. These patients at presentation and on follow-up did not receive anti-TB treatment.

All the laboratory staff performing the requested tests, including Xpert MTB/RIF and IFN- $\gamma$ measurement, were blinded to all microbiological and clinical information.

\section{IFN- $\gamma$ measurement}

Interferon gamma (IFN- $\gamma$ ) concentrations were measured in pleural fluid supernatant in duplicate using the InterGam Ultrasensitive Rapid Immuno-suspension Assay (IRISA; Antrum Biotech, Cape Town, South Africa; limit of detection $=5$ to $10 \mathrm{pg} / \mathrm{ml}$ ). Pleural fluid supernatant 
was prepared by centrifuging pleural fluid at $3000 \times \mathrm{g}$ for 15 min to remove any unwanted debris.

\section{Xpert MTB/RIF assay}

A $1 \mathrm{ml}$ aliquot of raw pleural fluid and a $1 \mathrm{ml}$ aliquot of concentrated (centrifuged) pleural fluid from each patient was diluted with $2 \mathrm{ml}$ of the Xpert MTB/RIF sample buffer. The $1 \mathrm{ml}$ concentrated pleural fluid aliquot was prepared by centrifugation of a median (IQR) of 20 (10-20) ml pleural fluid at $3000 \times$ g for $15 \mathrm{~min}$, with the supernatant discarded and the pellet made up to $1 \mathrm{ml}$ with phosphate buffer solution. The pleural fluid and sample buffer solutions were then mixed vigorously and incubated at room temperature for $15 \mathrm{~min}$, with further mixing halfway through the incubation. A $2 \mathrm{ml}$ volume of the diluted samples was then transferred to an Xpert MTB/RIF cartridge and run on the GeneXpert (Cepheid, Dx System Version 4.0c) machine. The limit of detection was determined in duplicate by spiking $0,50,75,100$ and $150 \mathrm{H} 37 \mathrm{Rv}$ CFU to $1 \mathrm{ml}$ aliquots of pleural fluid from subjects confirmed not to have TB, before dilution with sample buffer and subsequent Xpert MTB/RIF analysis. This experiment was repeated twice. Inhibition was evaluated by comparing the PCR cycle-threshold $\left(C_{T}\right)$ values of the internal positive control (lyophilized Bacillus atrophaeus subsp. globigii spores) from unconcentrated and concentrated samples.

\section{Statistical analysis}

Categorical variables were compared using the $x^{2}$ test and continuous variables were compared using Student's t-test where appropriate, with Mann-Whitney used for non-parametrically distributed continuous variables. Correlations were analyzed using the Spearman coefficient for non-parametrically distributed variables. Diagnostic accuracy, including 95\% confidence intervals, was assessed using sensitivity, specificity, positive predictive value (PPV), negative predictive value (NPV) (Open Epi, Version 2.3.1) and area under the receiver operator curve (AUROC) in definite-TB and non-TB groups (Graphpad Prism, Version 5.03).

\section{Results}

A total of 103 patients with pleural effusion and suspected TB were enrolled. Ten patients were excluded from the final analysis: three had incomplete clinical data and seven were on anti-TB treatment for more than 48 hours prior to the samples being taken (see Figure 1 for patient flow and test results available).

\section{Clinical and demographic data}

Forty subjects were confirmed to have definite-TB, 48 non-TB and 5 participants probable-TB (not meeting diagnostic criteria but receiving $\mathrm{TB}$ treatment). The subjects with non-TB effusions had a spectrum of malignant and non-malignant diagnoses including: lymphoma, adenocarcinoma, small cell carcinoma, and parapneumonic effusion. Of the 65 subjects consenting to HIV testing, $17 \%$ (11) were positive. Clinical and demographic data are summarized in Table 1.

\section{Performance outcomes of IFN- $\gamma$}

IFN- $\gamma$ levels were evaluated in 93 patients (40 definite$\mathrm{TB}, 5$ probable-TB and 48 non-TB). Median (IQR) IFN- $\gamma$ levels were approximately 100 times higher in definite-TB compared to non-TB effusions: 131.8 (131.8-162.7) versus 0.57 (0.0-5.71) pg/ml, $\mathrm{p}<0.0001$ (Figure 2). A receiver operating characteristic (ROC) curve derived rule-in cut point of $107.7 \mathrm{pg} / \mathrm{ml}$ was used to determine the sensitivity and specificity (95\% CI) (Figure 3). Table 2 compares the diagnostic accuracy of the IFN- $\gamma$ with other same-day diagnostics in definite-TB versus non-TB groups. IFN- $\gamma$ sensitivity was unaffected by the inclusion of probablewith definite-TB patients (see Additional file 1: Table S1 in the online supplementary data), and diagnostic accuracy was not significantly different in HIV-infected compared to uninfected patients (see Additional file 1: Table S2 in the online supplementary data).

\section{Performance outcomes of pleural fluid ADA}

Adenosine deaminase levels were measured in 84 patients. Median (IQR) ADA levels were 5 times higher in definite-TB compared to non-TB effusions: 51.7 (35.262.1) versus $11.5(7.1-18.8) \mathrm{U} / \mathrm{L}, \mathrm{p}<0.0001$. Using a clinical cut point of $>30 \mathrm{U} / \mathrm{L}$ (South African national standard [18]), the sensitivity (95\%CI), specificity, NPV and PPV were 79.0\% (63.7-89.0), 92.7\% (80.6-97.5), 82.7\% (69.391.0) and 91.0\% (73.4-95.4), respectively (Table 2). Relevant values using the rule-in cut-point (at least 95\% specificity) are shown in Table 2. The ROC and scatter plot of ADA are shown in Figures 2 and 3.

Grouping the probable TB with the definite-TB group reduced the sensitivity to $74.5 \%$ but the specificity remained unchanged, when using the clinically used cut point of $>30 \mathrm{U} / \mathrm{L}$. A similar trend occurred when using the rule-in cut point of $48.85 \mathrm{U} / \mathrm{L}$ (see Additional file 1: Table S1 in the online supplementary data). HIV status had no significant impact on the ADA diagnostic accuracy (see Additional file 1: Table S3 in the online supplementary data).

Performance outcome for Xpert MTB/RIF assay including inhibition and detection threshold

Xpert MTB/RIF was performed on 93 participants. Xpert MTB/RIF detected 9 out 40 subjects with definiteTB. The sensitivity $(95 \% \mathrm{CI})$ and specificity using $1 \mathrm{ml}$ of unprocessed pleural fluid was $22.5 \%(12.4,37.6)$ and 98.0\% (89.2, 99.7) respectively. Xpert MTB/RIF sensitivity 


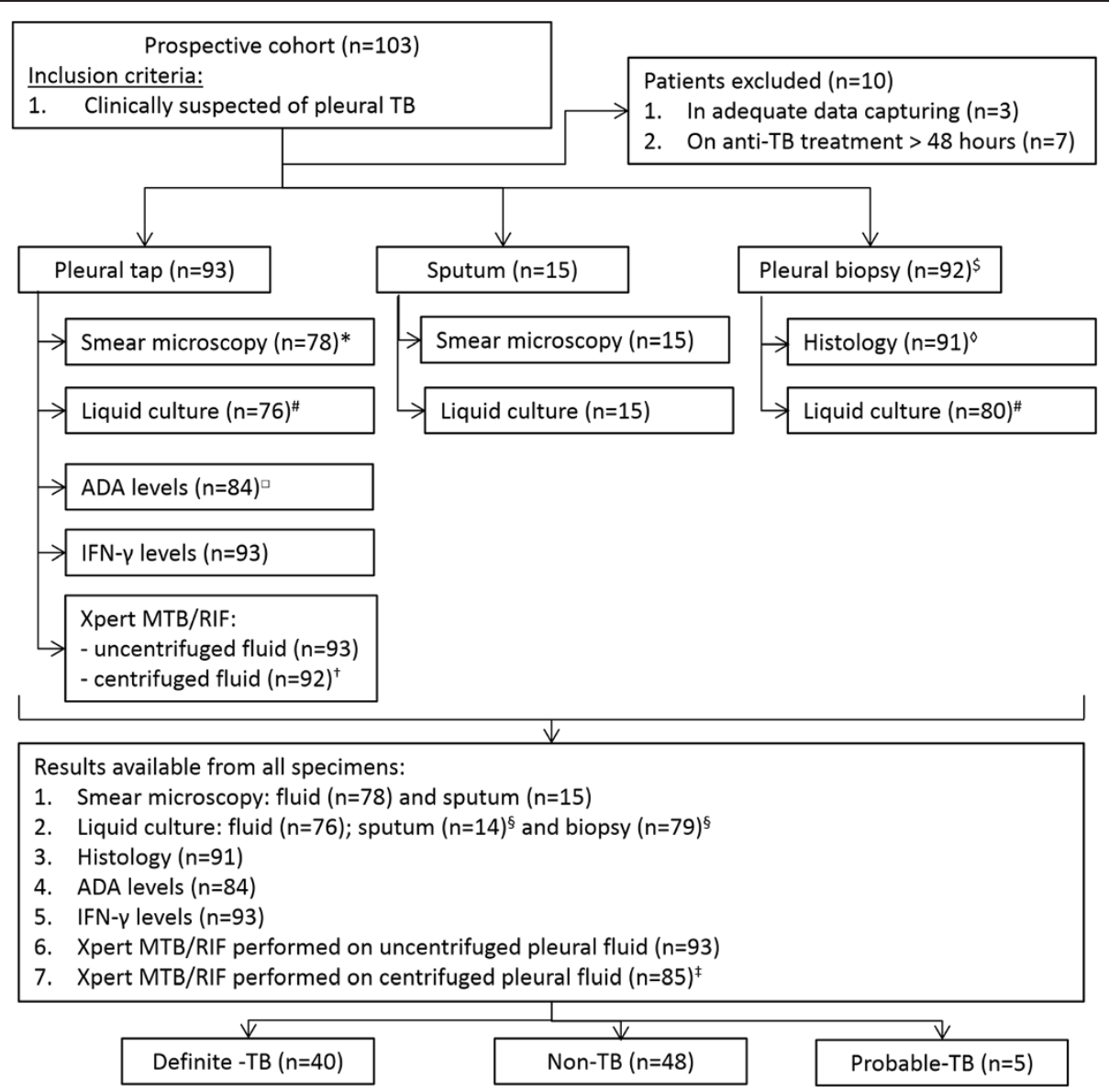

Figure 1 Patient flow and test results available for final diagnoses. ${ }^{\$}$ biopsy not performed $(n=1) ;{ }^{*}$ no fluid smear requested $(n=15) ;{ }^{*}$ liquid

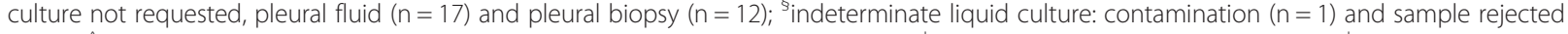
$(n=1)$; ${ }^{\circ}$ histology not requested $(n=1)$; ${ }^{\circ}$ ADA measurements not requested $(n=9)$; ${ }^{\dagger}$ sample lost during processing $(n=1),{ }^{*}$ indeterminate Xpert MTB/RIFF result $(n=7)$.

and specificity did not improve when a concentrated (centrifuged) pleural fluid samples was used $(\mathrm{p}=0.90$, Table 3$)$. However, the number of indeterminate results generated in concentrated compared to unprocessed pleural fluid samples was higher $7 / 92$ vs. $0 / 93(p<0.05)$. Table 2 compares the diagnostic accuracy of Xpert MTB/RIF and other same-day diagnostics. Of note, Xpert MTB/RIF pleural fluid testing detected one rifampicin-resistant result that was confirmed by liquid culture to be a truepositive MDR-TB case.

Spiking raw pleural fluid demonstrated that the Xpert MTB/RIF assay was able to reliably detect $\geq 75$ CFU per millilitre of pleural fluid (Figure 4a). No significant difference was seen between unprocessed and concentrated pleural fluid internal probe amplifications with median $\mathrm{C}_{\mathrm{T}}$ values of $(95 \% \mathrm{CI})$ of $27.6(27.5,28.5)$ and 27.4 (27.6, 28.9), respectively. The median time-to-positivity (TTP) (IQR) for pleural fluid and pleural biopsy culture were 28.0 days (23.3-31.1) and 19.0 days (17.7-24.6), respectively (data not shown). No correlation was observed between $\mathrm{C}_{\mathrm{T}}$ values and TTP from pleural fluid liquid culture, however, there was a correlation (Spearman $r=$ 0.93, $\mathrm{p}=0.02$ ) between $\mathrm{C}_{\mathrm{T}}$ values and the TTP from pleural biopsy culture.

In contrast to the findings with ADA and IFN- $\gamma$, when subjects were stratified by HIV status, Xpert MTB/RIF had a higher sensitivity in HIV infected versus uninfected persons [50\% (21.6-78.5) vs. 9.1\% (2.6-27.9), $\mathrm{p}=$ 0.031 (see Additional file 1: Table S4 in the online supplementary data). A similar trend was seen when comparing pleural fluid and biopsy culture as reference standard (see Additional file 1: Table S5 in the online supplementary data).

Xpert MTB/RIF in combination with ADA or IFN- $\gamma$ did not significantly improve the diagnostic accuracy when compared to ADA or IFN- $\gamma$ alone (Table 2).

\section{Discussion}

Given that a reliable same-day diagnostic tool for pleural TB is lacking, we prospectively evaluated and compared 
Table 1 Baseline characteristics of the definite tuberculosis (TB) and non-TB patients

\begin{tabular}{|c|c|c|c|}
\hline & Definite-TB & Non-TB & $p$-value \\
\hline Patients (n) & 40 & 48 & \\
\hline Age (yrs) & $39(29,55)$ & $61(54,69)$ & $<0 \cdot 0001^{\sharp}$ \\
\hline \multicolumn{4}{|l|}{ Sex } \\
\hline Male & $24(60 \%)$ & $29(60.4 \%)$ & \\
\hline Female & $16(40 \%)$ & $19(39.6 \%)$ & \\
\hline \multicolumn{4}{|l|}{ Race } \\
\hline White & $1(2.5 \%)$ & $5(10.4 \%)$ & \\
\hline Black & $28(70 \%)$ & $9(18.8 \%)$ & $<0.0001^{*}$ \\
\hline Mixed & $11(27.5 \%)$ & $34(70.8 \%)$ & $<0.0001^{*}$ \\
\hline \multicolumn{4}{|l|}{ HIV Status } \\
\hline HIV-infected & $8(20 \%)$ & $1(2.0 \%)$ & $<0.05^{*}$ \\
\hline HIV uninfected & $22(55 \%)$ & $29(60.4 \%)$ & \\
\hline Refused testing & $4(10 \%)$ & $3(6.3 \%)$ & \\
\hline Unknown & $6(15 \%)$ & $15(31.3 \%)$ & \\
\hline \multicolumn{4}{|c|}{ History of Previous TB } \\
\hline Yes & $6(15 \%)$ & $5(10.4 \%)$ & \\
\hline No & $31(77.5 \%)$ & $39(81.3 \%)$ & \\
\hline Unknown & $3(7.5 \%)$ & $4(8.3 \%)$ & \\
\hline \multicolumn{4}{|l|}{ BCG Status } \\
\hline Yes & $20(50 \%)$ & $25(52.1 \%)$ & \\
\hline No & $10(25 \%)$ & $5(10.4 \%)$ & \\
\hline Unknown & $10(25 \%)$ & $18(37.5 \%)$ & \\
\hline \multicolumn{4}{|l|}{ Current smoker } \\
\hline Yes & $7(17.5 \%)$ & $18(37.5 \%)$ & $<0.05^{*}$ \\
\hline No & $32(80 \%)$ & $26(54.1 \%)$ & $<0.05^{*}$ \\
\hline Unknown & $1(2.5 \%)$ & $4(8.3 \%)$ & \\
\hline
\end{tabular}

Data are presented as median (IQR) or $\mathrm{n}(\%)$ or yrs (IQR). BCG: bacilli Calmette-Guerin. " : unpaired t-test; *: Chi-squared test.

the use of ADA, IFN- $\gamma$ and Xpert MTB/RIF using pleural fluid from patients with suspected TB. Our key findings were (i) Xpert MTB/RIF had poor sensitivity and this was neither the result of a sub-optimal level of detection compared to other types of biological samples nor increased PCR inhibition, (ii) Xpert MTB/RIF sensitivity was not improved by concentrating larger volumes of pleural fluid, (iii) ADA and IFN- $\gamma$ are good rule-in tests for TB pleural effusions but the higher sensitivity of IFN- $\gamma$ would be preferable over ADA for rule-in, particularly in low-burden settings where a high INF- $\gamma$ level is unlikely to be due to cancer, (iv) the excellent rapid rule-out value of IFN- $\gamma$, compared to ADA, is of particular clinical usefulness in high burden settings as it could be used to prompt a search for an alternative diagnosis and hence medical or surgical biopsy. The sheer burden of disease and resource constraints, including lack of skilled operators, in high burden settings means that a routine biopsy-based approach cannot be undertaken. Moreover, the majority of patients suspected with pleural TB were unable to produce sputum.

The Xpert MTB/RIF assay, which can simultaneously detect the presence of $M$. tuberculosis and rifampicin resistance in sputum specimens, has shown great promise in the rapid diagnosis of $\mathrm{TB}$, with an average sensitivity and specificity of $90.4 \%$ and $98.4 \%$, respectively [8]. It has also improved the rapid diagnosis of pulmonary $\mathrm{TB}$ (sensitivity of $\sim 68 \%$ ) in smear-negative patients and recently been endorsed by the Scientific and Technical Advisory Board of the WHO for use in paucibacillary samples $[9,20]$. However, there are hardly any data about Xpert performance in pleural fluid. Published studies have generally been performed in low burden settings as a laboratory exercise reporting yields in samples from extra-pulmonary sites, and only reported on a handful of patients with TB pleural effusion [10,12-16]. This may explain the high sensitivity reported in these studies (range $58 \%$ to $100 \%$ ) and high specificities (87\% to $100 \%)$ [10-16]. Recently Friedrich and co-workers in a selected cohort found that 20 out of 25 patients had confirmed TB and that Xpert sensitivity was 25\% [11]. We have confirmed these findings in a larger unselected cohort and further interrogated the technical performance of the assay. The level of detection was $\geq 75$ colony forming units per $\mathrm{ml}$, and there was no evidence of PCR inhibition using the internal positive control. Centrifugation of pleural fluid made little difference. The time to positivity data (median $>28$ days) confirms the low organism load within the pleural space compared to pleural tissue. Indeed, there was a trend to shorter time to positivity in liquid culture from samples that were culture positive/Xpert positive compared to those that were culture positive/Xpert negative (data not shown). Collectively, these data confirm the notion that pleural TB is a paucibaciliary disease and thus concentration/ centrifugation of at least $20 \mathrm{ml}$ of pleural fluid makes little difference to diagnostic yield. Xpert performed better in HIV-infected individuals, which may reflect a higher organism load in the pleural fluid [21].

In high burden settings such as South Africa, a high ADA level is frequently used to guide initiation of antiTB therapy $[4,5]$. In this study, although ADA levels were 5 times higher in TB patients than in non-TB patients, using the laboratory accepted cut-point in Cape Town (30 U/L) roughly $20 \%$ of TB patients would have been missed and 1 in 10 incorrectly started on anti-TB therapy. Despite the inability of ADA to definitively confirm M. tuberculosis, it is a low cost and relatively rapid (same day) assay and has a high PPV when disease prevalence is high [22]. In low prevalence settings, however, PPV is too low to be clinically useful. Indeed, a recent meta-analysis of 63 studies including 2796 pleural TB 


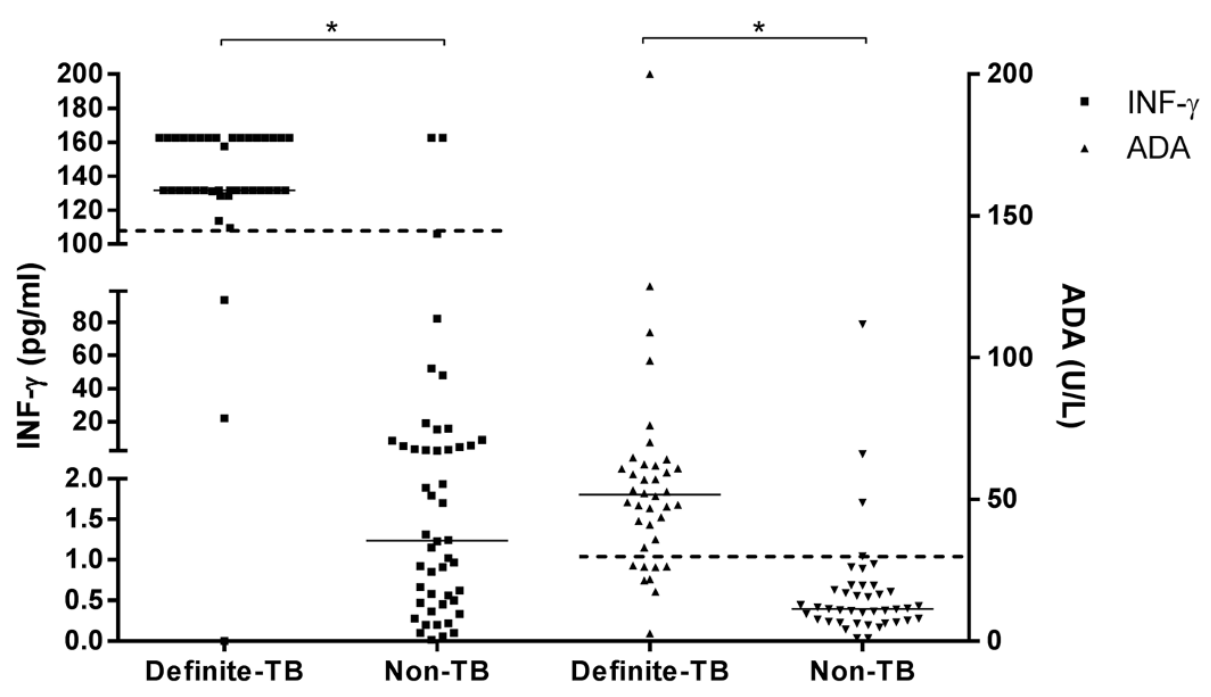

Figure 2 Scatter plot of interferon gamma (IFN- $\gamma$ ) and adenosine deaminase (ADA) using pleural fluid from patients with TB and non-TB pleural effusions. *: $p<0.0001$ (Mann-Whitney). See Additional file 1: Table S6 in the online supplementary data for final diagnosis of non-TB patients with ADA and/or IFN- $\gamma$ levels above cut points.

patients and 5297 non-TB patients reported the sensitivity of ADA to be $92 \%$ and specificity $90 \%$ at a cut-point of 41.9 U/L (median cut-point in the pooled studies, which each used a different cut-point) [4]. Thus, 1 in 10 patients would be over diagnosed with TB. The specificity of ADA may be improved if the proportion of lymphocytes is taken into account as was recently shown [22]. However, this is not universally helpful as about $\sim 25 \%$ of TB pleural effusions , may be neutrophil predominant [23].

Interferon gamma, similarly, was significantly higher in TB (100 fold) compared to non-TB patients. Using an ROC curve-determined cut-point of $107.7 \mathrm{pg} / \mathrm{ml}$, only 3 definite-TB patients were missed. By contrast, Xpert sensitivity, like pleural fluid culture $(\sim 40 \%)$ [2], was poor

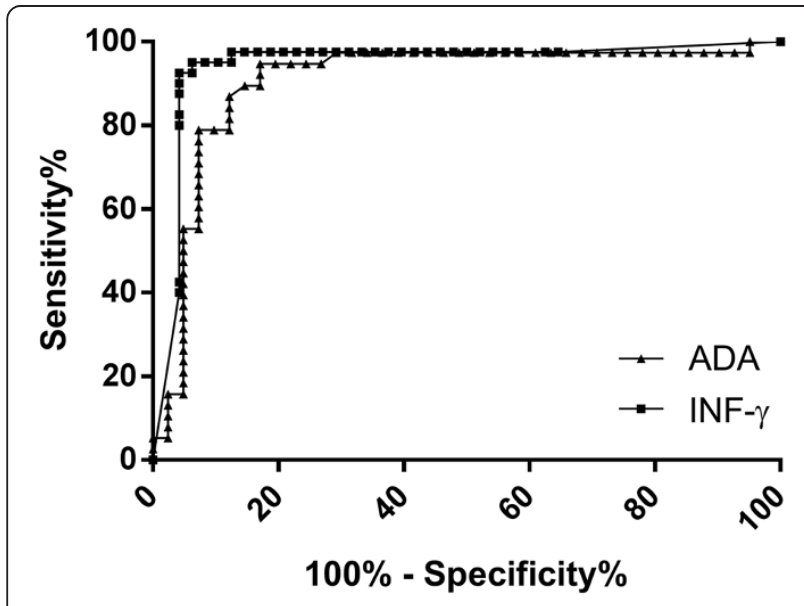

Figure 3 Area under the receiver operator characteristic (ROC) curves for interferon gamma (IFN- $\gamma$ ) and adenosine deaminase (ADA) were 0.94 and 0.91 , respectively. and therefore additional tools are required for optimal diagnosis when a non-biopsy approach is used. Thus, a 'one size fits all' approach with Xpert is inappropriate. Although closed pleural biopsy has a high diagnostic yield it is often unavailable in resource-poor settings, and even when available the large numbers of cases preclude routine biopsy in district general hospitals. In this study we have confirmed our previous findings, and those of others, that IFN- $\gamma$ is both a highly accurate rule-in and rule-out diagnostic test for pleural TB [6,24-26], however it must be accepted that the $107.7 \mathrm{pg} / \mathrm{ml}$ cut point was generated using the current cohort and further prospective testing is required to confirm the high sensitivity and specificity. Although it can be easily measured by a commercially available ELISA-kit, it is not routinely performed due to the high cost and the kits only being available in a 96 well format, which lead to a considerable wastage of unused wells [27]. Interestingly, of the only two non-TB patients that had elevated IFN- $\gamma$, one patient had an empyema, which is known to cause elevated IFN- $\gamma$ and is a contraindication to using the test, and the other patient was lost to follow-up and thus the true TB status was unclear. A previous study from our group demonstrated a similar accuracy (sensitivity $97 \%$ specificity 98\%) [6]. An obvious drawback is the lack of susceptibility data, but in resource-poor settings culture confirmation with susceptibility can follow if there is a poor response to treatment. In any event a culture isolate or positive NAAT would be required to determine susceptibility- the yield of both are low in pleural TB. It would have been interesting to perform Xpert on the pleural tissue biopsies but technically this was not feasible due to the limited 
Table 2 Per patient diagnostic accuracy of Xpert MTB/RIF, IFN- $\gamma$, and ADA for the diagnosis pleural tuberculosis

\begin{tabular}{|c|c|c|c|c|c|c|}
\hline \multirow[t]{2}{*}{ Assay } & \multicolumn{6}{|c|}{ Definite-TB vs. Non TB } \\
\hline & Sensitivity\% (95\% Cl) & Specificity\% (95\% Cl) & PPV\% (95\% Cl) & NPV\% $(95 \% \mathrm{Cl})$ & +LR $(95 \% \mathrm{Cl})$ & -LR (95\% CI) \\
\hline Xpert MTB/RIF & $22.5 \%$ *\#ロ\$£ $(12.4,37.6) 9 / 40$ & $98 \%(89.2,99.7) 47 / 48$ & $90 \%(59.6,98.3) 9 / 10$ & $60.3 \%^{+\bullet \bullet \bullet}(49.2,70.4) 48 / 78$ & $10.8(1.43,81.65)$ & $0.79^{\text {coxs }_{i}}(0.67,0.94)$ \\
\hline IFN- $\gamma$ & $92.5 \% * 0(80.2,97.5) 37 / 40$ & $95.9 \%(86.1,98.9) 46 / 48$ & $94.9 \%(83.2,98.6) 37 / 39$ & $93.9 \%^{+\infty}(83.5,97.9) 46 / 49$ & $22.20(5.70,86.46)$ & $0.08^{\zeta \circlearrowright}(0.03,0.23)$ \\
\hline ADA (Clinical cut point) & $79 \%^{\Delta \# \times}(63.7,89) 30 / 38$ & $92.7 \%(80.6,97.5) 38 / 41$ & $91 \%(73.4,95.4) 30 / 33$ & $82.7 \% \neq \triangleright(69.3,91.0) 38 / 46$ & $10.79(3.59,32.47)$ & $0.23^{\circ}(0.12,0.42)$ \\
\hline ADA (Rule-in cut point) & $55.3 \%$ मОхє¥ $(39.8,69.9)$ 21/38 & $95.2 \%(83.9,98.7) 39 / 41$ & $91.4 \%(73.3,97.6) 21 / 23$ & $69 \cdot 7 \%^{\infty \Delta}(56.7,80.1) 39 / 56$ & $11.33(2.85,45.1)$ & $0.47^{\text {að̃ }}(0.33,0.67)$ \\
\hline $\begin{array}{l}\text { Xpert MTB/RIF followed by IFN- } \gamma \\
\text { if MTB/RIF-negative }\end{array}$ & $92.5 \%^{\$ €}(80.2,97.5) 37 / 40$ & $93.8 \%(83.2,97.9) 45 / 48$ & $92.5 \%(80.2,97.5) 37 / 40$ & $93.8 \% \bullet \bullet(83.2,97.9) 45 / 48$ & $14.80(4.93,44.43)$ & $0.08^{\S p}(0.03,0.24)$ \\
\hline $\begin{array}{l}\text { Xpert MTB/RIF followed by ADA } \\
\text { (clinical cut-point) if MTB/RIF-negative }\end{array}$ & $81.6 \%{ }^{£ \neq}(66.6,90.8) 31 / 38$ & $92.7 \%(80.6,97.5) 38 / 41$ & $91.2 \%(77.1,97) 31 / 34$ & $84.5 \%^{*}(71.3,92.3) 38 / 45$ & $11.15(3.70,33.49)$ & $0.20^{i}(0.10,0.39)$ \\
\hline$p$-value & 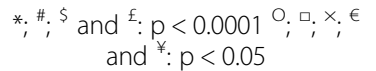 & & & $\begin{array}{c}{ }^{\dagger} \text { and } \bullet: P<0.0001^{\ominus} ;{ }^{\infty} ; \text {; } \\
\text { and }{ }^{\circ}: \mathrm{p}<0.05\end{array}$ & & 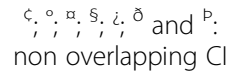 \\
\hline
\end{tabular}

A positive MTB (Mycobacterium tuberculosis) fluid culture and/or positive MTB biopsy culture and/or histology in keeping with MTB infection used as a reference. PPV: positive predictive value, NPV: negative predictive value, $+L R$ : positive likelihood ratio, $-L R$ : negative likelihood ratio, Cl: confidence interval, IFN- $\gamma$ : interferon gamma, $A D A$ : adenosine deaminase. IFN- $\gamma$ cut point of $107.7 \mathrm{pg} / \mathrm{ml}$ determined by ROC. ADA clinical cut point of $30 \mathrm{U} / \mathrm{L}$ is used for clinical decision-making at Groote Schuur hospital. Rule-in ADA cut point of 48.85U/l determined by ROC.

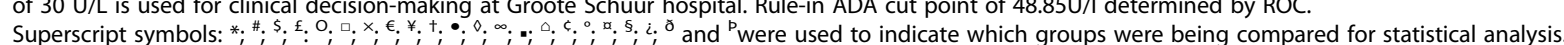


Table 3 Per patient diagnostic accuracy of Xpert MTB/RIF assay using centrifuged and uncentrifuged pleural fluid to diagnose pleural tuberculosis

\begin{tabular}{|c|c|c|c|c|}
\hline \multirow[t]{2}{*}{ Assay } & \multicolumn{4}{|c|}{ Definite-TB vs. Non-TB } \\
\hline & Sensitivity\% (95\% CI) & Specificity\% (95\% CI) & PPV\% $(95 \% \mathrm{CI})$ & NPV\% $(95 \% \mathrm{Cl})$ \\
\hline Pleural fluid (uncentrifuged) & $22.5 \%(12.4,37.6) 9 / 40$ & $98 \%(89.2,99.7) 48 / 48$ & $90 \%(59.6,98.3) 9 / 10$ & $60.3 \%(49.2,70.4) 48 / 78$ \\
\hline Centrifuged pellet & $23.7 \%(13.0,39.3) 9 / 38$ & $100 \%(91.7,100) 42 / 42$ & $100 \%(70.1,100) 9 / 9$ & $59.2 \%(48.2,70.3) 42 / 71$ \\
\hline p-value & $n s^{*}$ & $\mathrm{~ns}^{*}$ & $n s^{*}$ & $n s^{*}$ \\
\hline
\end{tabular}

amount of tissue and potential for tube blockage in the machine when solid material is used. However this may not have improved the diagnostic accuracy, in a recent study, Xpert was unable to detect any TB cases and more indeterminate results occurred when performed on finely ground pleural tissue [17].

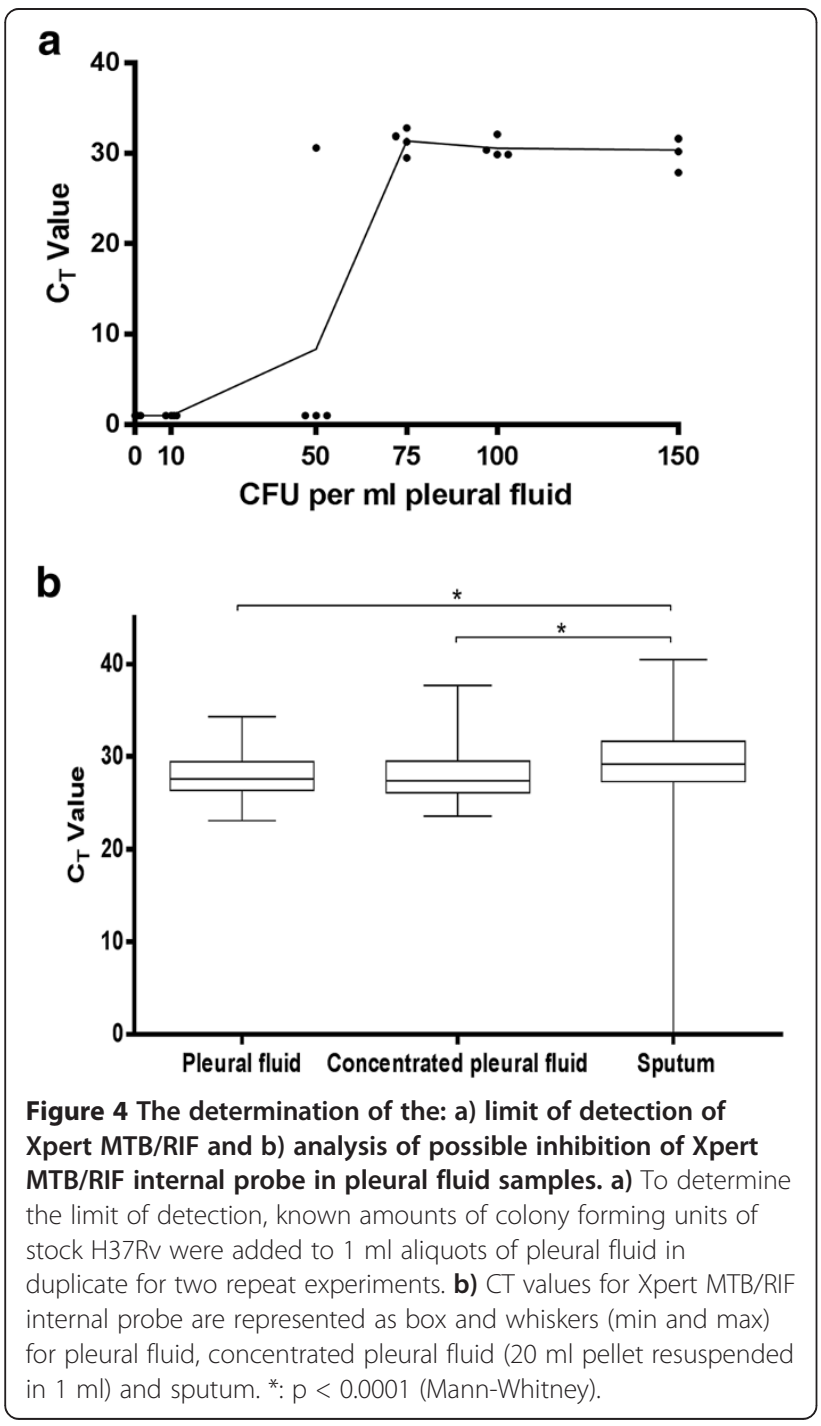

Affordability and cost effectiveness remains an important consideration in resource poor TB endemic countries. A comprehensive cost effectiveness analysis was beyond the scope of this paper, and we were unable to perform a simple cost analysis given the lack of a clinically validated commercially available unstimulated interferon gamma assay. Although the GeneXpert MTB/RIF assay is now being rolled out in many TB endemic countries [28], as we have demonstrated, sensitivity is largely sub-optimal $[6,29]$. Although ADA is widely available, specificity may also be also sub-optimal, as we and others have previously demonstrated. Nevertheless, it remains a widely available relatively low cost test. Diagnosing drug-resistant pleural TB also merits cost consideration. However, the GeneXpert MTB/RIF assay has a poor sensitivity in this context and thus whatever diagnostic modality is used (unstimulated interferon gamma, ADA, or GeneXpert MTB/RIF) pleural tissue or fluid culture is still required for susceptibility testing. There is an urgent need to make available a commercially and clinically validated, relatively rapid, single patient use assay for the measurement of unstimulated interferon gamma levels in pleural fluid and other forms of EPTB.

There are several limitations of our study. There were a low proportion of HIV-infected patients and several patients with unknown HIV status. However, HIV prevalence rates in TB patients in the Western Cape Province of SA are known to be lower than in the rest of the country [30], and patients often refuse testing. We only centrifuged $20 \mathrm{ml}$ of fluid. However, we were limited by available sample, particularly when effusions were loculated. Moreover, a recent paper has showed that increasing the pleural fluid volume to $100 \mathrm{ml}$ does not improve culture yields, despite improving the time to positivity [21]. The conclusions drawn here apply to high TB and HIV burden settings and, for the sake of external validity, require confirmation in other settings. However, given that HIV increases the concentration of organisms in pleural fluid and that the upper limit of the Xpert sensitivity $(95 \% \mathrm{CI})$ was $37 \%$ suggests that Xpert is unlikely to perform well in any setting when using pleural fluid. We did not evaluate the potential impact on 
morbidity and length of hospital stay of ADA, IFN- $\gamma$ and Xpert compared to empiric treatment based on laboratory analysis alone (lymphocyte predominance), however, this would have require a randomized design and up to $\sim 25 \%$ of TB effusions are known to be neutrophil predominant [23]. The confidence intervals of the sensitivity estimates for interferon gamma and the GeneXpert MTB/ RIF assay are not ideal and therefore our findings should be confirmed using larger sample numbers and from different parts of the world.

\section{Conclusion}

The clinical usefulness of Xpert-MTB/RIF to diagnose pleural TB is limited by its poor sensitivity. By contrast, IFN- $\gamma$ is an excellent rule-in test and, compared to ADA, has significantly better sensitivity and rule-out value in a high HIV prevalence setting. The high NPV of IFN- $\gamma$, compared to ADA, is particularly useful to clinicians as it prompts further work-up and tissue biopsy in patients who are unlikely to have TB, however further prospective testing is required.

\section{Additional file}

Additional file 1: Table S1. Per patient diagnostic accuracy of Xpert MTB/RIF, IFN- $\gamma$, and ADA for the diagnosis pleural tuberculosis. Table S2. Per patient diagnostic accuracy of IFN- $\gamma$ for the diagnosis of pleural tuberculosis, stratified by HIV status. Table S3. Per patient diagnostic accuracy using ADA for the diagnosis of pleural tuberculosis, stratified by HIV status. Table S4. Per patient diagnostic accuracy of the Xpert MTB/ RIF assay, stratified by HIV status. Table S5. Per sample diagnostic accuracy of the XpertMTB/RIF assay for the diagnosis of pleural tuberculosis, using either fluid or biopsy culture as reference standard. Stratified by HIV status. Table S6. Non-TB patients with ADA and/or INF- $\gamma$ levels above specified cut points.

\section{Competing interests}

KD, UG and GT have performed consultancy work for Antrum Biotech (Pty) Ltd, a UCT co-owned spin-off company, and kits for the study were donated by the company. However, Antrum Biotech played no role in study design, data analysis or its publication. Similarly, KD has been the recipient of grant funding from FIND Diagnostics, who also donated Xpert MTB/RIF cartridges to carry out the study but FIND played no role in study design, data analysis or its publication.

\section{Authors' contributions}

Concept and design, KD, RM, GT, JP. Laboratory experiments, RM, AB. Analysis and interpretation, RM, RvZS, JP, GC, GS, GN, BA, HK. Drafting the manuscript for important intellectual content, KD, RM, RvZS, GT, JP, UG. All authors read and approved the final manuscript.

\section{Acknowledgements}

This work was supported by the South African National Research Foundation (SARChl; KD), and the European and Developing Countries Clinical Trials Partnership (EDCTP; TB-NEAT and TESA; KD and GT).

Received: 20 December 2013 Accepted: 3 April 2014

Published: 8 April 2014

\section{References}

1. World Health Organization: WHO: Global Tuberculosis Report 2012. Geneva, Switzerland: WHO; 2012:100.
2. Light RW: Update on tuberculous pleural effusion. Respirology 2010, 15(3):451-458.

3. Koegelenberg CF, Diacon AH: Pleural controversy: close needle pleural biopsy or thoracoscopy-which first? Respirology 2011, 16(5):738-746.

4. Liang QL, Shi HZ, Wang K, Qin SM, Qin XJ: Diagnostic accuracy of adenosine deaminase in tuberculous pleurisy: a meta-analysis. Respir Med 2008, 102(5):744-754.

5. Jiang J, Shi HZ, Liang QL, Qin SM, Qin XJ: Diagnostic value of interferongamma in tuberculous pleurisy: a metaanalysis. Chest 2007, 131(4):1133-1141.

6. Dheda K, van Zyl-Smit RN, Sechi LA, Badri M, Meldau R, Meldau S, Symons G, Semple PL, Maredza A, Dawson R, Wainwright H, Whitelaw A, Vallie Y, Raubenheimer P, Bateman ED, Zumla A: Utility of quantitative T-cell responses versus unstimulated interferon-\{gamma\} for the diagnosis of pleural tuberculosis. Eur Respir J 2009, 34(5):1118-1126.

7. Theron G, Peter J, van Zyl-Smit R, Mishra H, Streicher E, Murray S, Dawson R, Whitelaw A, Hoelscher M, Sharma S, Pai M, Warren R, Dheda K: Evaluation of the Xpert MTB/RIF assay for the diagnosis of pulmonary tuberculosis in a high HIV prevalence setting. Am J Respir Crit Care Med 2011, 184(1):132-140.

8. Chang K, Lu W, Wang J, Zhang K, Jia S, Li F, Deng S, Chen M: Rapid and effective diagnosis of tuberculosis and rifampicin resistance with Xpert MTB/RIF assay: a meta-analysis. J Infect 2012, 64(6):580-588.

9. World HealthOrganization: Report of the Tenth Meeting WHO Strategic and Technical Advisory Group for Tuberculosis (STAG-TB). Geneva: World Health Organization; 2010:27-29.

10. Causse M, Ruiz P, Gutierrez-Aroca JB, Casal M: Comparison of two molecular methods for rapid diagnosis of extrapulmonary tuberculosis. J Clin Microbiol 2011, 49(8):3065-3067.

11. Friedrich SO, von Groote-Bidlingmaier F, Diacon AH: Xpert MTB/RIF assay for diagnosis of pleural tuberculosis. J Clin Microbiol 2011, 49(12):4341-4342.

12. Hillemann D, Rusch-Gerdes S, Boehme C, Richter E: Rapid molecular detection of extrapulmonary tuberculosis by the automated GeneXpert MTB/RIF system. J Clin Microbio/ 2011, 49(4):1202-1205.

13. Miller MB, Popowitch EB, Backlund MG, Ager EP: Performance of Xpert MTB/RIF RUO assay and IS6110 real-time PCR for Mycobacterium tuberculosis detection in clinical samples. J Clin Microbiol 2011 , 49(10):3458-3462.

14. Moure R, Martin R, Alcaide F: Effectiveness of an integrated real-time PCR method for detection of the Mycobacterium tuberculosis complex in smear-negative extrapulmonary samples in an area of low tuberculosis prevalence. J Clin Microbiol 2012, 50(2):513-515.

15. Tortoli E, Russo C, Piersimoni C, Mazzola E, Dal Monte P, Pascarella M, Borroni E, Mondo A, Piana F, Scarparo C, Coltella L, Lombardi G, Cirillo DM: Clinical validation of Xpert MTB/RIF for the diagnosis of extrapulmonary tuberculosis. Eur Respir J 2012, 40(2):442-447.

16. Vadwai V, Boehme C, Nabeta P, Shetty A, Alland D, Rodrigues C: Xpert MTB/RIF: a new pillar in diagnosis of extrapulmonary tuberculosis? J Clin Microbiol 2011, 49(7):2540-2545.

17. Christopher DJ, Schumacher SG, Michael JS, Luo R, Balamugesh T, Duraikannan P, Pollock NR, Pai M, Denkinger CM: Performance of Xpert (R) MTB/RIF on pleural tissue for the diagnosis of pleural tuberculosis. Eur Respir J 2013, 42(5):1427-1429.

18. Blake J, Berman P: The use of adenosine deaminase assays in the diagnosis of tuberculosis. S Afr Med J 1982, 62(1):19-21.

19. Boonyagars LKS: Use of Adenosine Deaminase for the Diagnosis of Tuberculosis: A Review. J Infect Dis Antimicrob Agents 2010, 27:8.

20. Steingart KR, Sohn H, Schiller I, Kloda LA, Boehme CC, Pai M, Dendukuri N: Xpert (R) MTB/RIF assay for pulmonary tuberculosis and rifampicin resistance in adults. Cochrane Database Syst Rev 2013, 1:1-29.

21. von Groote-Bidlingmaier F, Koegelenberg CF, Bolliger CT, Chung PK, Rautenbach C, Wasserman E, Bernasconi M, Friedrich SO, Diacon AH: The yield of different pleural fluid volumes for Mycobacterium tuberculosis culture. Thorax 2013, 68(3):290-291.

22. Garcia-Zamalloa A, Taboada-Gomez J: Diagnostic accuracy of adenosine deaminase and lymphocyte proportion in pleural fluid for tuberculous pleurisy in different prevalence scenarios. PloS one 2012, 7(6):e38729.

23. McGrath EE, Warriner D, Anderson PB: Pleural fluid characteristics of tuberculous pleural effusions. Heart Lung 2010, 39(6):540-543. 
24. Hiraki A, Aoe K, Eda R, Maeda T, Murakami T, Sugi K, Takeyama H: Comparison of six biological markers for the diagnosis of tuberculous pleuritis. Chest 2004, 125(3):987-989.

25. Liu YC, Shin-Jung Lee S, Chen YS, Tu HZ, Chen BC, Huang TS: Differential diagnosis of tuberculous and malignant pleurisy using pleural fluid adenosine deaminase and interferon gamma in Taiwan. J Microbiol Immunol Infect 2011, 44(2):88-94.

26. Wang H, Yue J, Yang J, Gao R, Liu J: Clinical diagnostic utility of adenosine deaminase, interferon-gamma, interferon-gamma-induced protein of $10 \mathrm{kDa}$, and dipeptidyl peptidase 4 levels in tuberculous pleural effusions. Heart Lung 2012, 41(1):70-75.

27. Sharma SK, Banga A: Pleural fluid interferon-gamma and adenosine deaminase levels in tuberculosis pleural effusion: a cost-effectiveness analysis. J Clin Lab Anal 2005, 19(2):40-46.

28. Theron G, Zijenah L, Chanda D, Clowes P, Rachow A, Lesosky M, Bara W, Mungofa S, Pai M, Hoelscher M, Dowdy D, Pym A, Mwaba P, Mason P, Peter J, Dheda K: Feasibility, accuracy, and clinical effect of point-of-care Xpert MTB/RIF testing for tuberculosis in primary-care settings in Africa: a multicentre, randomised, controlled trial. Lancet 2014, 383(9915):424-435.

29. Maartens G, Bateman ED: Tuberculous pleural effusions: increased culture yield with bedside inoculation of pleural fluid and poor diagnostic value of adenosine deaminase. Thorax 1991, 46(2):96-99.

30. Mayosi BM, Wiysonge CS, Ntsekhe M, Volmink JA, Gumedze F, Maartens G, Aje A, Thomas BM, Thomas KM, Awotedu AA, Thembela B, Mntla P, Maritz F, Blackett KN, Nkouonlack DC, Burch VC, Rebe K, Parish A, Sliwa K, Vezi BZ, Alam N, Brown BG, Gould T, Visser T, Shey MS, Magula NP, Commerford PJ. Clinical characteristics and initial management of patients with tuberculous pericarditis in the HIV era: the Investigation of the Management of Pericarditis in Africa (IMPI Africa) registry. BMC Infect Dis 2006, 6:2.

doi:10.1186/1471-2466-14-58

Cite this article as: Meldau et al:: Comparison of same day diagnostic tools including Gene Xpert and unstimulated IFN- $\gamma$ for the evaluation of pleural tuberculosis: a prospective cohort study. BMC Pulmonary Medicine 2014 14:58

\section{Submit your next manuscript to BioMed Central and take full advantage of:}

- Convenient online submission

- Thorough peer review

- No space constraints or color figure charges

- Immediate publication on acceptance

- Inclusion in PubMed, CAS, Scopus and Google Scholar

- Research which is freely available for redistribution 